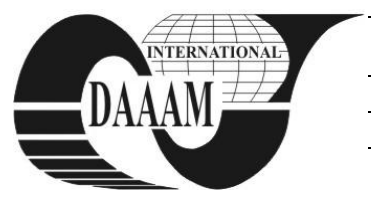

\title{
ESTABLISHING THE LONGITUDINAL ELASTICITY MODULUS OF RECOVERABLE SILICONE RUBBER
}

\section{POPESCU, D[aniel]; BUZATU, D[umitru] S[tefan]; GAVRILA, R[aluca] \& POPESCU, M[arian] C[atalin]}

Abstract: The paper presents the main elements that define some of the dynamic properties of recoverable silicone rubber. There are presented the variations of the storage modulus ( $\left.E^{\prime}\right)$ and loss modulus (E") as well as the methods used to determine them.

Key words: dynamic properties, silicone rubber, storage modulus, loss modulus

\section{INTRODUCTION}

Due to its properties, recoverable silicone rubber obtained through specific technologies is the most stable polymer currently available, offering good reliability combined with long life at convenient production costs. It offers the best resistance/time ratio both in normal and extreme conditions.

Applications of silicone rubber are not new in precision engineering and medical domain. Recovered silicone rubber is cheap to obtain and using it offers dynamic properties at least $\mathrm{s}$ good as the original material, especially in case of sensor technique tools and measuring tools.

\section{VISCOELASTIC PROPERTIES RECOVERABLE SILICONE RUBBER}

OF

It is known the fact that viscoelastic materials such as silicone rubber present both viscous and elastic properties. If a traction strain is applied to a viscoelastic material, the specific deformation can be determined by a differential equation of the form:

$$
\sigma=E \varepsilon+\mu \frac{d \varepsilon}{d t}
$$

Where:

$\sigma-$ traction effort unit

$\mathrm{E}$ - represents the elastic deformation in which the energy is stored as potential energy and used for returning to initial shape

$\mu$ - represents the viscous flow of the system in which the energy is dissipated by heat when the strain ends.

If $\mathrm{E}$ and $\mu$ are constants of depend on time, then (1) represents an ordinary linear differential equation. Considering:

$$
\begin{aligned}
& \sigma(t)=\sigma_{0} e^{i w t} \\
& \varepsilon(t)=\varepsilon_{0} e^{i w t}
\end{aligned}
$$

It results:

$$
\varepsilon_{0}=\frac{\sigma_{0}}{E+i \mu \omega}
$$

And also:

$$
\varepsilon=\frac{\sigma_{0} e^{i \omega t}}{E+i \mu \omega}=\frac{\sigma}{E+i \mu \omega}
$$

The denominator from (5) can be expressed in terms like:

Where:

$$
E=E^{\prime}+i E^{\prime \prime}
$$

$$
\begin{aligned}
& \text { E - complex modulus } \\
& E^{\prime}-\text { storage modulus } \\
& E^{\prime \prime}=\omega \mu-\text { loss modulus }
\end{aligned}
$$

Relation (5) becomes:

$$
\varepsilon=\frac{\sigma}{E}
$$

The complex module:

$$
|E|=\sqrt{E^{2}+E^{\prime \prime 2}}
$$

Also, the phase difference $(\sigma, \varepsilon)$ is given by:

$$
\delta=\operatorname{tg}^{-1} \frac{E^{\prime \prime}}{E^{\prime}}
$$

The variation $\sigma=f(\varepsilon)$ in case of a viscoelastic material (sillicone) is presented in fig. 1. The graph indicates the fact that this type of behavior applies to very small deformations.

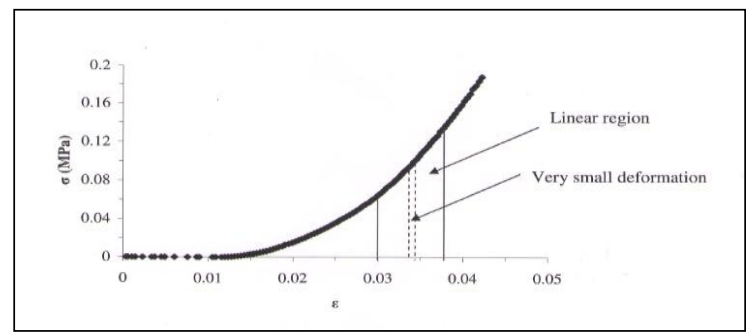

Fig. 1. Linear viscoelastic behavior of silicone

In case of recoverable silicone rubber the existence of the loss modulus emphasizes the hysteresis presence, in which the strain variation $\sigma=f(\varepsilon)$ does not follow the same path (fig. 2).

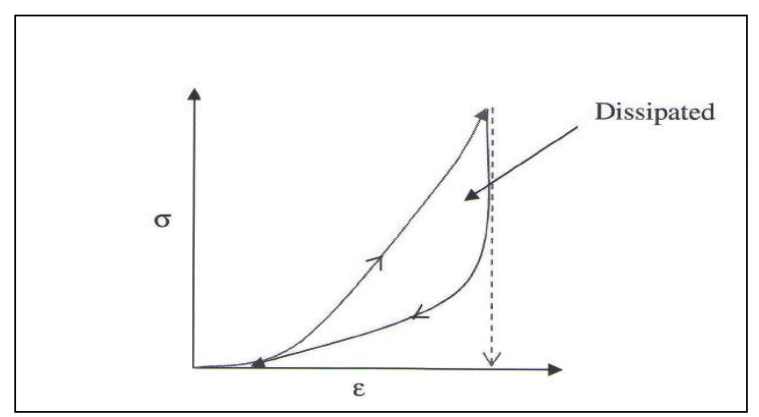

Fig. 2. Hysterezis phenomenon for silicone rubber 


\section{DYNAMIC ANALYSIS OF RECOVERABLE SILICONE RUBBER}

Determining the variation of E' and E" represents the most popular method for studying the viscoelastic behavior of polymers.

In this case there are applied sinusoidal strains and is measured the specific deformation of the material, allowing the determination of the dynamic modulus $E$ through its components.

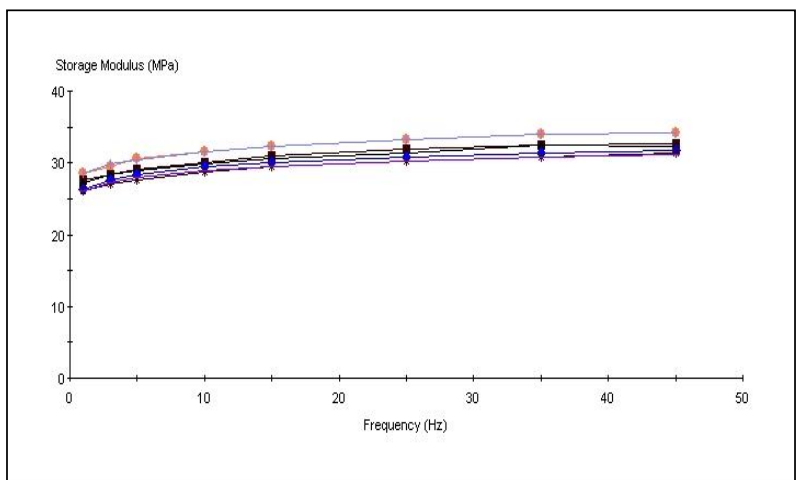

Fig. 3. Storage modulus variation

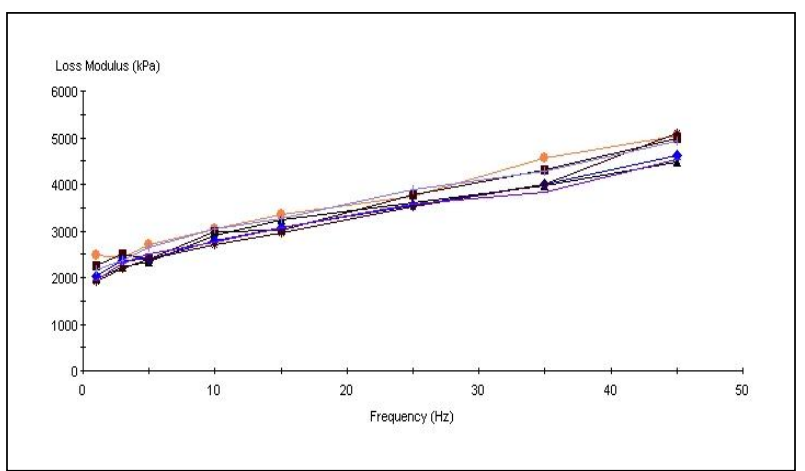

Fig. 4. Loss modulus variation

\section{CONCLUSIONS}

- The technological products for which the above elasticity modulus was obtained present various shapes ranging from simple to complex (fig. 5, 6).

- It was also used a technological product obtained by molding $100 \%$ liquid silicone rubber.

- The other products were obtained from $40 \%$ recovered silicone rubber powder with $25 \mu \mathrm{m}-1.5 \mathrm{~mm}$ granularity and $60 \%$ liquid silicone rubber.

- In similar load conditions, storage modulus and load modulus were measured at different frequencies; the temperature was maintained constant at $\mathrm{T}=20^{\circ}$.

- Recoverable silicone rubber belongs to a category of polymers formed by long molecular chains, presenting unique viscoelastic properties and a combination of elastic solids and Newtonian fluids characteristics.

- The following table presents a comparison of the medium elasticity modulus in case of recoverable silicone rubber versus normal rubber:

\begin{tabular}{|l|l|}
\hline Rubber & $\mathrm{E}=0.002 \mathrm{GPa}$ \\
\hline Recoverable silicone rubber & $\mathrm{E}=\mathbf{0 . 0 3 5} \mathbf{~ G P a}$ \\
\hline
\end{tabular}

Tab.1. Elasticity modulus comparison

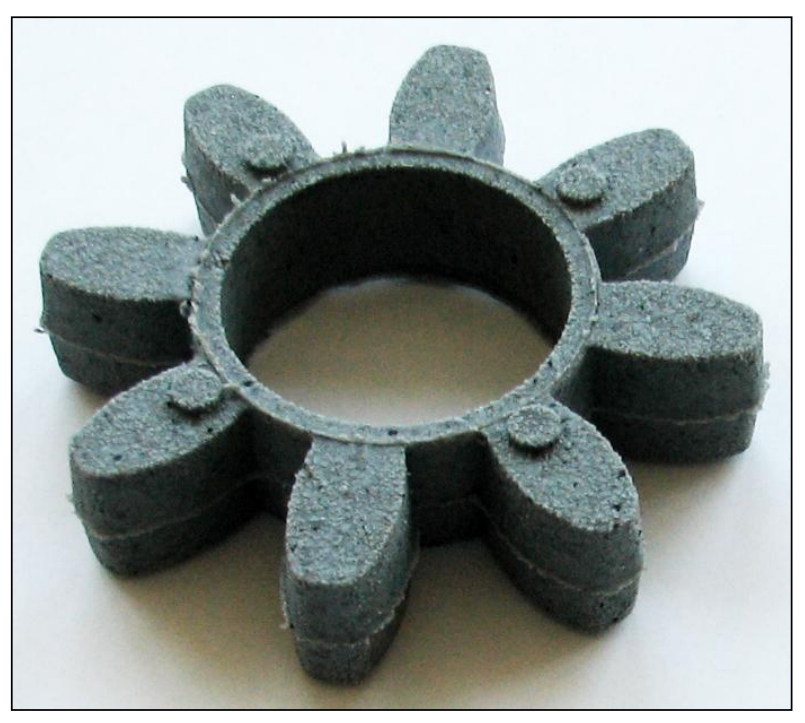

Fig. 5. Elastic coupling - octagonal profile evolvent gear; $60 \%$ recovered silicone rubber powder, $40 \%$ liquid silicone rubber

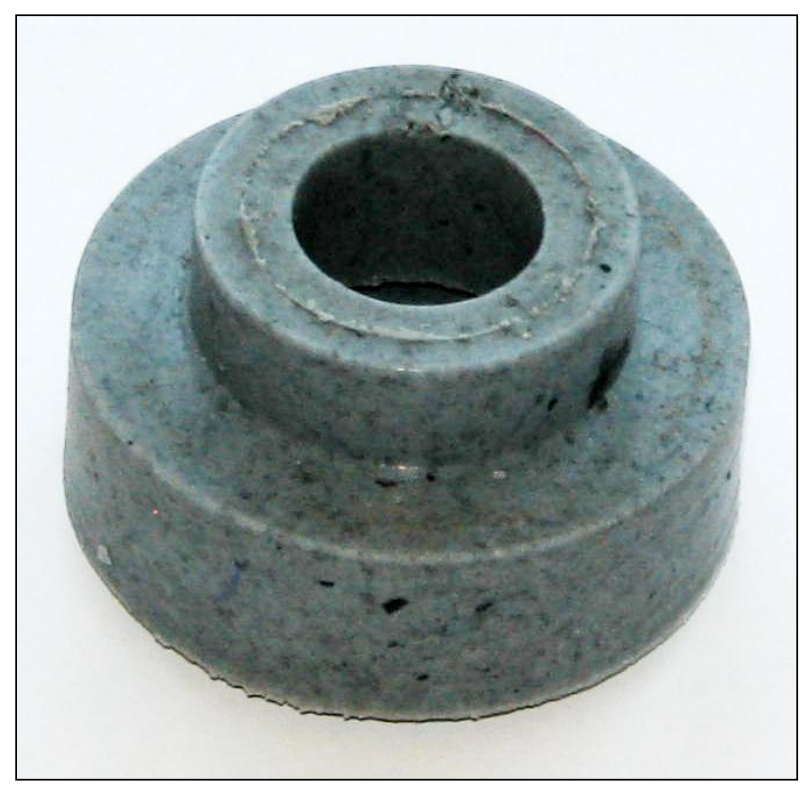

Fig. 6. Semi-coupling; 60\% recovered silicone rubber powder, $40 \%$ liquid silicone rubber

\section{REFERENCES}

Aziz, T.; Waters, M. \& Jagger, R. (2003). Analysis of the properties of silicone rubber maxillofacial prosthetic materials, Journal of Dentistry 31, pp. 67-74

Huba, A.; Molnár, L.; Czmerk, A. \& Fischl, T. (2005). Dynamic Analysis of Silicone Elastomers, Journal Materials Science Forum, vol. 473 - 474, pp. 85-90

Mahomed, A. (2008) Properties of Elastomers for Small Joint Replacements, PhD Thesis, University Of Birmingham, 2008

Popescu, D.; Buzatu, St. \& Gavrila, R. (2010). Study Of Surface Roughness At Finishing Of Recovered Silicone Rubber, The $21^{\text {th }}$ International Daaam Symposium "Intelligent Manufacturing \& Automation: Focus On Interdisciplinary Solutions", ISSN 1726-9679, pg. 00050007, Zadar, Croatia

*** (2008) Technologies for recovering and using material from composite insulators with external rubber coating. R\&D contract within MEF sectorial research and development plan 